\title{
Maria Teresa Diez Grieser und Roland Müller: Mentalisieren mit Kindern und Jugendlichen Hrsg. von Ulrich Schultz-Venrath (Klett-Cotta, Stuttgart, 2018)
}

\author{
Rosmarie Barwinski (Winterthur)
}

Das Buch «Mentalisieren mit Kindern und Jugendlichen» von Maria Teresa Diez Grieser und Roland Müller ist der 4. Band einer Reihe, herausgegeben von Ulrich Schultz-Venrath, die Anwendungsmöglichkeiten mentalisierungsbasierter Massnahmen auf wichtigen Störungsfeldern aufzeigt. Die Thematik «Mentalisieren in Gruppen» wurde im ersten Band dargelegt. Ein zweiter Band fokussierte sich auf «Mentalisieren bei Depressionen». Eine Abhandlung zum Thema «Mentalisieren bei Somatisierungsstörungen» in einem dritten Band ist noch in Vorbereitung.

Die Autorin und der Autor zeigen sehr praxisnah auf, wie Mentalisieren bei Kindern und Jugendlichen gefördert werden kann. Ob Kinder die Fähigkeit, das eigene Verhalten und das Verhalten anderer Menschen durch Zuschreibung mentaler Zustände zu interpretieren, erwerben können, hängt wesentlich von der Bindungserfahrung eines Kindes oder Jugendlichen ab. «Mentalisieren erzeugt Mentalisieren - Nichtmentalisieren erzeugt Nichtmentalisieren». Das Kind benötigt dazu die Erfahrung von sicheren Bindungen. Eine zentrale Rolle spielen deshalb die Eltern, weshalb im Buch nicht nur die mentalisierungsbasierte Psychotherapie mit Kindern vorgestellt wird, sondern auch die Elternarbeit als wichtig erachtet und folglich detailliert besprochen wird.

Entsprechend ist das Buch aufgebaut. Nach der Einleitung folgt eine theoretische Einführung, die Mentalisieren als zentrale Entwicklungsaufgabe darstellt. Es wird beschrieben, wie wir uns die Entwicklung des Mentalisierens vorstellen können. Prämentale Modi und Mentalisieren werden definiert. Neu ist, dass neben den von Fonagy und seiner Arbeitsgruppe empirisch abgeleiteten prämentalen Modi (teleologischer Modus, psychischer Äquivalenzmodus und Als-ob-Modus) ein Körpermodus ergänzt wird, der die Bedeutung des Körpers und die körperliche Entwicklung im ersten Lebensjahr für die Entwicklung der Fähigkeit zu mentalisieren miteinbezieht.

Im dritten Kapitel steht «Mentalisieren als zentrale elterliche Fähigkeit» im Fokus. Die Bedeutung von «epistemischem Vertrauen» - «die Bereitschaft eines 
Individuums, neues Wissen von Seiten einer anderen Person als vertrauenswürdig, generalisierbar und relevant für das eigene Selbst einzustufen» (S. 53) - und wie der Bindungsstil der Eltern dieses fördern oder auch beeinträchtigen kann, wird anschaulich vermittelt.

Wie Mentalisieren in der Kinder- und Jugendlichen-Psychotherapie gefördert werden kann, ist Thema des vierten Kapitels. Prinzipien der therapeutischen Mentalisierungsförderung bei Kindern und Jugendlichen sowie die Förderung eines mentalisierenden Klimas in der Familie werden anhand von Fallbeispielen anschaulich illustriert. Es wird deutlich, dass es nicht primär um die Anwendung von Techniken geht, sondern es ist die mentalisierende Haltung der/des Therapeutin/ en, die als zentral erachtet wird.

Das fünfte Kapitel ist der «Praxis der therapeutischen Mentalisierungsförderung» gewidmet. Hier wird Schritt für Schritt beschrieben, wie konkret in einer mentalisierungsbasierten Psychotherapie bei Kindern und Jugendlichen vorgegangen werden sollte: Die Phase der Abklärung, die Arbeit am Mentalisieren in der Mittelphase sowie die Phase der Beendigung der Therapie.

Im sechsten Kapitel werden mentalisierungsstärkende Interventionen mit Müttern/Eltern und Kleinkindern in verschiedenen Settings vorgestellt.

Im siebten Kapitel steht «Mentalisieren in pädagogischen Kontexten und in der Prävention» im Vordergrund. Das Buch schliesst mit einem Fazit und Ausblick.

Das Buch überzeugt durch seine gelungene Verknüpfung zwischen Theorie und Praxis. Vor allem der praktische Teil vermittelt sehr nachvollziehbar, wie man sich mentalisierungsbasierte Psychotherapie vorstellen kann, was sie auszeichnet und welche Veränderungsschritte mit diesem Ansatz gefördert oder möglich gemacht werden können. Mentalisieren wird als Prozess verstanden, der über eine bestimmte Kompetenz oder ein Entwicklungsniveau hinausreicht und deshalb von besonderer Relevanz für die Konstruktion von Bedeutung innerhalb der psychoanalytischen Situation ist.

Das Buch wendet sich an PsychotherapeutInnen, aber auch an Fachpersonen, die im pädagogischen Kontext mit Kindern und Jugendlichen arbeiten, die Verhaltensauffälligkeiten aufgrund von Entwicklungsdefiziten und/oder durch Traumata aufweisen. 